\title{
La Leucémie Myéloïde Chronique Pédiatrique: Une Entité Très Rare Au Service d'Hématologie De Yopougon
}

\author{
Kouakou B., \\ Conde A., \\ Packo DSS, \\ Kamara I., \\ Silué DA, \\ Botti RP, \\ Djeket R., \\ Koffi $K G$, \\ Sanogo I.,
}

Service d'hématologie clinique,

Centre Hospitalier Universitaire de Yopougon, Abidjan Côte d'Ivoire

\section{Résumé}

Contexte: La leucémie myéloïde chronique (LMC) est un syndrome myéloprolifératif dû à une prolifération myéloïde monoclonale prédominant sur la lignée granuleuse. Son pronostic a été amélioré par l'avènement des inhibiteurs de la tyrosine kinase. Elle survient le plus souvent chez l'adulte jeune. Les auteurs rapportent un cas clinique chez un enfant de 6 ans. Présentation de cas: Il s'agissait d'un enfant de 6 ans, de sexe masculin, référé en consultation en hématologie pour splénomégalie volumineuse évoluant depuis 3 mois. L'hémogramme a montré une hyperleucocytose à 282 Giga/L avec myélémie importante et polymorphe une anémie à $66 \mathrm{~g} / \mathrm{l}$ et une thrombocytose à $870 \mathrm{G} / \mathrm{L}$. L'examen cytogénétique a retrouvé le chromosome Philadelphie sans anomalie additionnelle. Le traitement par imatinib mesylate a pu être débuté. Conclusion: La leucémie myéloïde chronique est certes rare chez l'enfant mais les praticiens doivent y penser devant une hyperleucytose importante persistente.

Mots clés: Leucémie Myéloïde Chronique, Enfant, Rare, Yopougon 


\title{
Pediatric Chronic Myeloid Leukemia an Uncommon Entity at the Haematology Clinic of Yopougon
}

\author{
Kouakou B., \\ Conde A., \\ Packo DSS, \\ Kamara I., \\ Silué DA, \\ Botti RP, \\ Djeket R., \\ Koffi $K G$, \\ Sanogo I.,
}

Service d'hématologie clinique,

Centre Hospitalier Universitaire de Yopougon, Abidjan Côte d'Ivoire

\begin{abstract}
Background: Chronic myeloid leukemia (CML) is a myeloproliferative syndrome due to monoclonal myeloid proliferation predominant over the granular line. His prognosis was improved by the advent of tyrosine kinase inhibitors. It occurs most often in young adults. The authors report the clinical case of a 6-year-old child because of its rarity. Case report: This was a 6-year-old male child, referred in hematology consultation for persistent of large splenomegaly. The hemogram showed hyperleucocytosis at $282 \mathrm{Giga} / \mathrm{L}$ with large myelemia and polymorphic anemia at $66 \mathrm{~g} / \mathrm{l}$ and thrombocytosis at $870 \mathrm{G} / \mathrm{L}$. the cytogenetic analyse found the Philadelphia chromosome without additional anomaly. The treatment with imatinib mesylate has therefore begun. Conclusion: Although the CML is uncommon at young people, but practicians must think about it when we have an important hyperleucocytosis.
\end{abstract}

Keywords: Chronic Myeloid Leukemia, Child, Uncommun, Yopougon

\section{Introduction}

La leucémie myéloïde chronique (LMC) est un syndrome myéloprolifératif dû à une prolifération myéloïde monoclonale sans blocage de maturation, prédominant sur la lignée granuleuse. Il s'agit de la première 
pathologie directement corrélée à une anomalie cytogénétique acquise clonale (Amine, 2017).

Cette anomalie a été mise en évidence dès 1960 sous la forme d'un chromosome anormal appelé le chromosome de Philadelphie, qui se produit par translocation réciproque entre les chromosomes 9 et 22 . La découverte de cette anomalie chromosomique a permis au chercheur de développer une thérapie ciblée de cette maladie qui est l'inhibiteur de la tyrosine kinase. La LMC de l'enfant et de l'adolescent est peu fréquente et représente moins de 3\% des leucémies dans ce groupe d'âge (Amine, 2017 ; Fall, Ndiaye, Dior et al. 2015).

Les auteurs rapportent le cas de leucémie myéloïde chronique chez un enfant de 6 ans qui est le plus jeune patient suivi pour LMC dans le service à ce jour afin de préciser la particularité de la prise en charge.

\section{Observation}

Il s'agissait d'un petit garçon de 6 ans, $3^{\text {ème }}$ enfant d'une fratrie de 4, accouché par voie basse, sans antécédents particuliers, emmené en consultation par ses parents pour splénomégalie volumineuse, une hyperleucocytose à $282 \mathrm{Giga} / \mathrm{L}$ avec anémie à $66 \mathrm{~g} / \ell$ et une thrombocytose à $870 \mathrm{G} / \mathrm{L}$.

L'histoire de sa maladie remonterait à trois mois par l'apparition d'une asthénie physique, d'une fièvre à répétition sans foyer infectieux associée à une distension abdominale sans trouble digestif. Après plusieurs consultations dans les structures sanitaires où un traitement est initié sans succès, l'enfant est adressé au service d'hématologie pour une meilleure prise en charge.

L'examen physique notait un état général moyen, des conjonctives pâles et une splénomégalie de type IV de Hackett. Les aires ganglionnaires périphériques étaient sans particularité.

$\mathrm{Au}$ plan paraclinique, l'hémogramme montrait une hyperleucocytose à $225 \mathrm{G} / \mathrm{L}$, une anémie microcytaire hypochrome à $87 \mathrm{~g} / \ell$ et une thrombocytose à $708 \mathrm{G} / \mathrm{L}$. Le frottis périphérique notait une myélémie importante et polymorphe. Le myélogramme réalisé dans les suites montrait une perturbation de l'équilibre entre les lignées en faveur de la lignée granuleuse et la présence de myélocytes hyper granuleux compatible avec une LMC en phase chronique. La symptomatologie évoluait dans un contexte de fléchissement. L'examen physique notait des conjonctives pâles et une splénomégalie de type IV de Hackett. L'analyse cytogénétique sur la moelle osseuse dans le cadre du bilan étiologique confirmait la présence du chromosome Philadelphie qui était la translocation $\mathrm{t}(9 ; 22)$ entre le bras long d'un chromosome 9 en q34 et le bras d'un chromosome 22 en q11. Toutes les mitoses analysées présentent un caryotype pseudodiploïde (nombre modal à 46 chromosomes) avec la translocation $\mathrm{t}(9 ; 22)$. Il n'y avait pas d'autre 
mutation supplémentaire. La biologie moléculaire n'a pu être faite car à la charge du patient qui ne disposait pas de couverture sociale.

Il n'y a pas eu de prise d'hydroxyurée avant la confirmation du diagnostic et la mise en route de l'Imatinib. Le bilan biochimique multiparamétrique montrait le taux de LDH augmenté à 500 UI/L alors que l'ionogramme sanguin, l'urémie, la créatininémie de même que l'hémostase de routine étaient normaux. L'échographie abdominale réalisée confirmait la splénomégalie homogène dont la taille est estimée à $13 \mathrm{~cm}$. L'enfant est hospitalisé avec début d'un traitement symptomatique par hyperhydratation, en vue de la prévention du syndrome de lyse.

L'Imatinib (inhibiteur de la tyrosine kinase de 1ère génération) à 340 $\mathrm{mg} / \mathrm{m}^{2} /$ jour par voie orale a pu être débuté à la confirmation du diagnostic. On notait une rémission hématologique avec une normalisation de l'hémogramme. L'évolution était marquée par une amélioration de l'état général avec une régression de la splénomégalie, qui est passée de stade IV à II après 2 semaines de traitement par l'Imatinib. Sur le plan biologique, une diminution du nombre des globules blancs qui s'était normalisé complètement au bout de 2 semaines de traitement spécifique et avec une bonne tolérance au traitement, marquée par une augmentation progressive de l'appétit. Il n'y avait pas d'effet secondaire signalé. L'évaluation à un mois confirmait la persistance de la rémission hématologique. L'évaluation moléculaire est envisagée à trois mois du suivi.

\section{Discussion}

La LMC de l'enfant est peu fréquente et représente moins de 3\% des leucémies (Amine, 2017). La maladie est très rare, surtout avant l'âge de 6 ans. Une étude faite au Sénégal par Fall et al (2015) chez les enfants montrait un âge médian de 14 ans avec un extrême de 9 à 17 ans (Smith, Ries, Gurney et al, 1999). Amine (2017) au Maroc avait trouvé un âge médian de 11 ans avec un extrême de 2 à 15 ans. Ce cas est le plus jeune patient diagnostiqué porteur de leucémie myéloïde chronique dans le service.

Compte tenu du caractère exceptionnel, la présentation clinique était symbolisée par une splénomégalie importante stade IV selon Hackett associée à une altération de l'état général. En effet la LMC survient le plus souvent chez un adulte jeune en bon état général. Chez l'enfant, son caractère exceptionnel expliquerait son retard diagnostic. Ce qui pourrait aussi justifier la découverte d'une hyperleucocytose à $225 \mathrm{G} / \ell$ avec myélémie importante et polymorphe, une anémie et thrombocytose. La présentation clinicobiologique de la LMC pédiatrique est différente de celle de l'adulte. Le nombre médian de globules blancs (GB) au départ dans la LMC chez l'adulte varie de 80 à 150 G/L (Suttorp, Eckardt, Tauer, et al, 2012). Cependant, chez les enfants, le nombre médian était de $250 \mathrm{G} / \mathrm{L}$ dans une étude de 200 patients d'âge médian 
de 11,6 ans. L'analyse des données d'une étude randomisée sur la LMC a montré que les adolescents et les jeunes adultes avaient une caractéristique clinique évocatrice de maladie agressive avec une plus grande taille de la rate, un nombre de leucocytes supérieur, avec un pourcentage élevé de blastes périphériques et hémoglobine inférieure à la normale comparés aux autres groupes d'âge (Pushpam \& Bakhshi, 2019).

Concernant le diagnostic, le chromosome Philadelphie était positif avec présence de translocation $\mathrm{t}(9 ; 22)$ sans mutation supplémentaire. Selon la littérature, la présence d'anomalie chromosomique supplémentaire est un facteur pronostic péjoratif majeur.

Pour le traitement, le patient avait bénéficié d'un traitement spécifique par thérapie ciblée (Imatinib) avec une réponse clinique et hématologique en 2 semaines. Selon la littérature, l'Imatinib est le médicament de première ligne, efficace qui entraîne à 3 mois une rémission hématologique de $95 \%$ et à 12 mois une rémission cytogénétique complète de $93 \%$ et une réponse moléculaire majeure de 85\% (Suttorp et al., 2012 ; Kantarjian, Dixon, Keating et al, 1988). Toutefois, l'arrêt de l'Imatinib après une rémission moléculaire complète prolongée n'est pas encore recommandé chez les enfants (Hijiya, Millot, Suttorp ; 2015).

Par rapport à l'effet secondaire de l'Imatinib, le patient tolérait bien le médicament. Selon la littérature, l'Imatinib est bien tolérée avec peu d'effet secondaire chez l'enfant mais son utilisation nécessite une grande vigilance vue l'absence de recommandation précise sur son utilisation et la durée du traitement qui est pour le moment à vie. Parmi les effets secondaires signalés sont les nausées, vomissements, diarrhée, hépatopathie, crampe musculaire, douleur osseuse et articulaire, décélération de la croissance, des lésions osseuses et un dysfonctionnement de la thyroïde. Les auteurs recommandent la surveillance du calcium, du phosphore, d'hormone thyroïdienne et parathyroïdienne, de vitamine $\mathrm{D}$ à six semaines du début du traitement, suivi tous les six mois (Pushpam \& Bakhshi, 2019 ; Kantarjian et al.,1988 ; Hijiya et al., 2015).

\section{Conclusion}

La LMC chez l'enfant est très rare. Il s'agit du premier plus jeune cas de LMC dans le service. La manifestation clinique est plus bruyante et il y a une hyperleucocytose très élevée par rapport à celle des adultes. L'Imatinib est le traitement spécifique standard avec une bonne réponse clinique, hématologique et cytologique. Pourtant, il faut bien surveiller les risques d'apparition des effets secondaires, surtout à long terme, qui ne sont pas encore connus pour le moment.

Conflit d'intérêt : Les auteurs ne déclarent aucun conflit d'intérêt. 


\section{Contribution des auteurs :}

Dr Kouakou Boidy, Dr Condé Abdoulaye, Dr Packo Dieu-le-veut Saint-Cyr Sylvestre : principaux investigateurs ont effectué la recherche documentaire et la rédaction de l'article.

Dr Kamara Ismaël, Dr Silué Dohoma Alexis, Dr Botti Renée-Paule, Dr Djeket Ruth ont participé au suivi de l'enfant au Service d'hématologie clinique du chu de Yopougon.

Pr Koffi Kouassi Gustave et Pr Sanogo Ibrahima ont participé à l'encadrement scientifique de ce travail.

\section{References:}

1. Amine H. La leucémie myéloïde chronique chez l'enfant : expérience du Service d'Hématologie et Oncologie pédiatrique de Rabat. Maroc. 2017. Thèse Médecine $n^{\circ} 38.142$ pages.

2. Fall S., Ndiaye F.S.D, Dior O.D. et al. Leucémie myéloïde chronique des sujets jeunes: expérience dans une unité d'hématologie Clinique au Sénégal. Revue d'Oncologie hématologie pédiatrique. 2015; 3 (4) :170-4.

3. Smith M.A, Ries L.A.G, Gurney J.G., Ross J.A. Cancer Incidence and Survival among Children and Adolescents: United States SEER Program 1975-1995. Leukemia, p17-34

4. Suttorp M., Eckardt L., Tauer T. J, et al. Management of chronic myeloid leukemia in childhood. Curr Hematol Malig Rep. 2012;7 (2):116-24.

5. Pushpam D., Bakhshi S. Paediatric chronic myeloid leukemia: Is it really a different disease? Indian J Med Res. 2019; 149: 600-9.

6. Kantarjian HM, Dixon D, Keating MJ et al. Characteristics of accelerated disease in chronic myelogenous leukemia. Cancer. 1988; 61: 1441-6.

7. Hijiya N, Millot F, Suttorp M. Chronic myeloid leukemia in children: Clinical findings, management and unanswered questions. Pediatr Clin North Am. 2015;62 : 107-19. 